\title{
Effects of Particle Stacking Angle on Heat Transfer Characteristics of Particles Close to the Wall
}

\section{Kai Zhang, Peng Sun*, Bin Zheng, Jiguo Xu, Youtang Wang, Zhenling Wang, Quanzhen Wang, Yongqi Liu}

School of Transportation and Vehicle Engineering, Shandong University of Technology, Zibo, China

Email: ${ }^{\star}$ zllsunpeng@sina.com

How to cite this paper: Zhang, K., Sun, P., Zheng, B., Xu, J.G., Wang, Y.T., Wang, Z.L., Wang, Q.Z. and Liu, Y.Q. (2021) Effects of Particle Stacking Angle on Heat Transfer Characteristics of Particles Close to the Wall. World Journal of Engineering and Technology, 9, 83-91.

https://doi.org/10.4236/wjet.2021.91006

Received: December 31, 2020

Accepted: February 2, 2021

Published: February 5, 2021

Copyright $\odot 2021$ by author(s) and Scientific Research Publishing Inc. This work is licensed under the Creative Commons Attribution International License (CC BY 4.0).

http://creativecommons.org/licenses/by/4.0/

\begin{abstract}
The primary energy demand increases, but a large amount of waste heat resources were not effectively used. To explore the influence of particle stacking structure on waste heat recovery process, CFD method was used to simulate. An unsteady heat transfer model of two particles was established, effect of particle stacking angle on heat transfer characteristics of the particles close to the wall under different initial temperature conditions was studied. Results show that: higher initial temperature, resulting in increased heat transfer time, the larger particle stacking angle causes the shortening of heat transfer time. When initial temperature is $1073 \mathrm{~K}$, the average wall heat flux shows a trend of rapid decline first and then a slow one. At the same moment, the larger stacking angle causes smaller particle average temperature. The change of particle stacking angle shows a greater impact on the temperature of the particles close to adiabatic wall. The increase in the stacking angle resulting in better heat transfer characteristics between particles.
\end{abstract}

\section{Keywords}

Stacking Structure, Waste Heat Recovery, Stacking Angle, Particles Close to the Wall, Numerical Simulation

\section{Introduction}

The rapid growth of the global economy has promoted the rapid development of the industrial field, which increases the consumption of primary energy. China has low energy efficiency and high energy consumption per unit of GDP, which is 2.5 times the world average. In 2019, China's total primary energy production was 3.97 million tons, while the total energy consumption was 4.87 million tons 
[1]. The total production is much smaller than the total consumption. Thus, energy saving and consumption reduction have become one of the important measures to solve the low energy efficiency.

A large number of high-temperature solid particles are produced in the industrial field [2]. If these particles are directly discharged, it will cause environmental pollution and energy waste. Therefore, the heat recovery technology and principles of high-temperature solid particles have received widespread attention. Most of the solid particles are irregular in shape and rough surface. The particles rub and collide with each other during the accumulation process, resulting in the positions of two adjacent particles constantly change, as well as the stacking structure of the particles. In actual production, high-temperature solid materials are randomly stacked in the packed bed. The irregularities of the stacked structure and the diversity of particle sizes and shapes make the research move hard. Thus, the research on the heat transfer characteristics of the packed bed from regular arrangement [3] to irregular arrangement [4], from single particle size [5] to irregularly shaped particles [6], from the simplified numerical model [7] to the actual production [8].

Porosity [9] is the most commonly used parameter to discuss particle stacking structure, but it is not comprehensive. The thickness of the bed, the particle stacking angle, and the effective contact number are important factors that affect the stacking structure. In a packed bed with uniform particle size, the change in the stacking angle between two particles is the main reason for the changes in other factors.

Therefore, an unsteady heat transfer model of two particles near the wall is established by CFD method in this paper, considering the influence of initial temperature, and the effect of particle stacking angle change on average wall heat flux and particle temperature at the initial temperature of $1073 \mathrm{~K}$ is studied. The results may provide basic theoretical support for the high-temperature solid particle waste heat recovery technology.

\section{Description of Model}

Figure 1 shows four typical particle stacking structures. The blue particles are in direct contact with the yellow particles. Affected by the stacking structure, there is an angle between the stacking direction of the two particles and the overall heat transfer direction. Smooth sphere was adopted to establish model. Factors affecting heat transfer such as particle extrusion deformation, surface roughness, and viscous gas are comprehensively considered. Bu's method [7] was adopted, a short cylinder is added at the contact position between particles to simulate the contact thermal resistance. Two particles close to the wall were selected as the research objects (stacking angle range $0^{\circ}-75^{\circ}$ ). After the above simplification, the physical model is shown in Figure 2.

The fluidity of gas in the packed bed is poor, Zheng's method [5] was adopted, the convection heat transfer was ignored, only the influence of heat conduction 


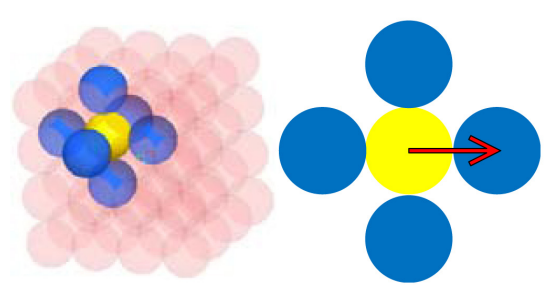

(a)

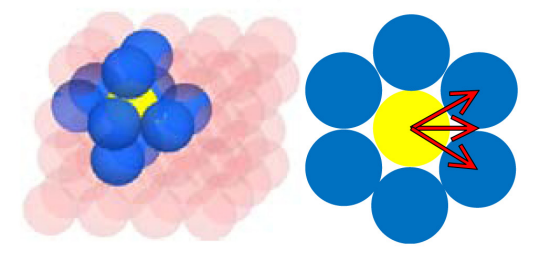

(c)

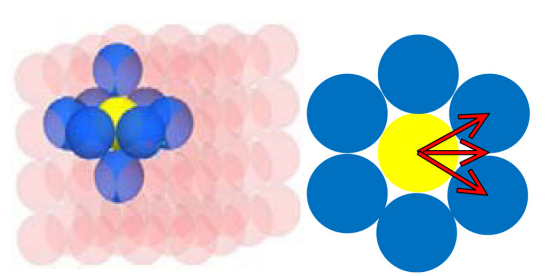

(b)

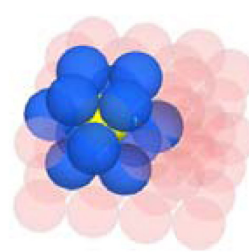

(d)

Figure 1. Typical stacking structure and particle stacking angle. (a) simple cubic stack; (b) oblique cube stack; (c) wedge tetrahedron stack; (d) rhombohedron stack.

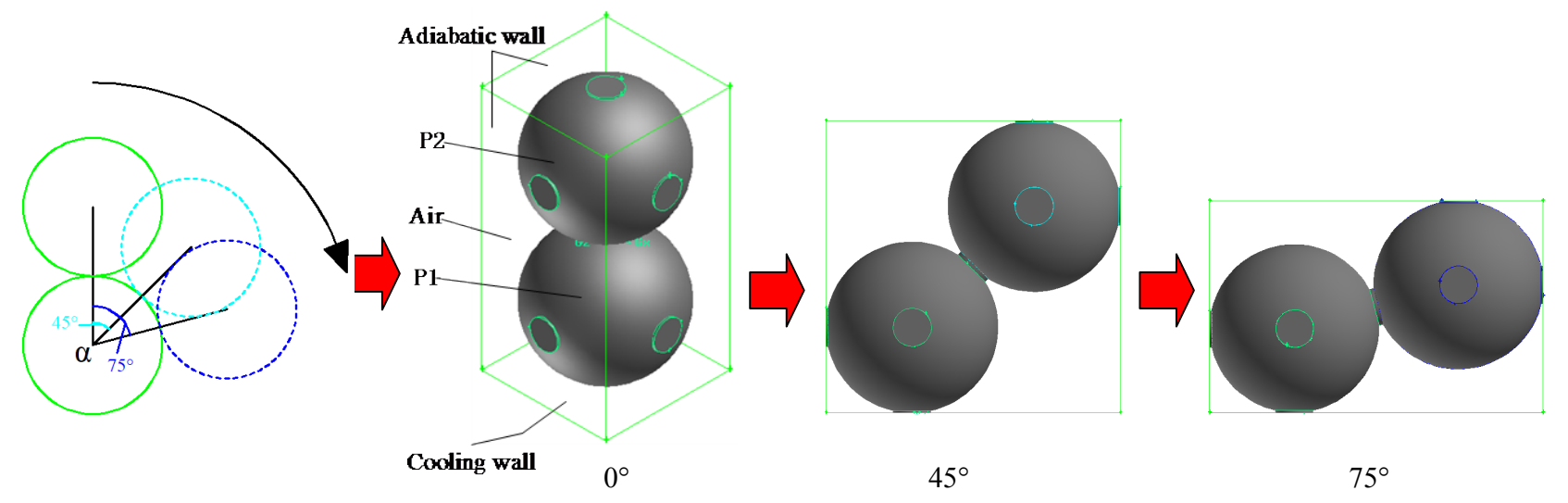

Figure 2. physical model.

radiation was considered. DO model is used to simulate the radiation heat transfer. The radiation heat transfer is calculated into the source term $\left(S_{t}\right)$. The heat conduction and radiation equations can be expressed as:

1) Heat condition equation

$$
\rho c \frac{\partial T}{\partial t}=\lambda\left(\frac{\partial^{2} T}{\partial x^{2}}+\frac{\partial^{2} T}{\partial y^{2}}+\frac{\partial^{2} T}{\partial z^{2}}\right)+S_{t}
$$

2) Radiation equation

$$
\begin{gathered}
\boldsymbol{s} \cdot \nabla I=0 \\
I\left(r_{w} \cdot \boldsymbol{s}\right)=\varepsilon_{w} \frac{\sigma T^{4}}{\pi}+\left(1-\varepsilon_{w}\right) \frac{q_{i n}}{\pi} \\
q_{\text {in }}=\int_{\boldsymbol{s} \cdot \boldsymbol{n}>0} I_{i n} \boldsymbol{s} \cdot \boldsymbol{n} \mathrm{d} \Omega
\end{gathered}
$$

In the formula, $\rho$ is Density, $\mathrm{kg} \cdot \mathrm{m}^{-3} ; T$ is Temperature, $\mathrm{K} ; t$ is time, $\mathrm{s} ; \lambda$ is Thermal conductivity, $\mathrm{W} \cdot \mathrm{m}^{-1} \cdot \mathrm{K}^{-1} ; S_{t}$ is Radiation item; $s$ is Radiation direction; $I$ is Partial surface radiation intensity, $\mathrm{W} \cdot \mathrm{m}^{-2} \cdot \mathrm{sr}^{-1} ; \varepsilon_{w}$ is Surface emissivity of 
the particle; $q_{\text {in }}$ is Particle surface radiation heat flow, $\mathrm{W} \cdot \mathrm{m}^{-2} ; \boldsymbol{n}$ is Unit normal vector; $\Omega$ is Spatial angle, sr.

The solid phase is meshed before gas phase, to ensure accurate and fast calculation results. The overall grid and local encryption are shown in Figure 3. The established physical model is imported into ANSYS Fluent for calculation. Initial conditions are shown in Table 1. Calculations are performed until 2.5\% of initial enthalpy value.

In this paper, effect of the initial temperature condition on the heat transfer time $\left(t_{h t}\right)$ is considered. The initial temperature is $1073 \mathrm{~K}$ is selected, the average wall heat flux $(\bar{q})$ and particle temperature $\left(\bar{T}, \bar{T}_{\mathrm{P} 1}, \bar{T}_{\mathrm{P} 2}\right)$ are studied, the values under different heat transfer times are selected as the evaluation criteria. The average wall heat flux is defined by Equation (5).

$$
\bar{q}=Q_{t} / A_{t}
$$

In the formula, $\bar{q}$ is Average wall heat flux, $\mathrm{W} \cdot \mathrm{m}^{-2} ; Q_{t}$ is Total heat transfer rate, $\mathrm{W} ; A_{t}$ is Heat transfer area, $\mathrm{m}^{-2}$.

\section{Results and Discussion}

\subsection{Heat Transfer Time}

Figure 4 shows the effect of particle stacking angle on heat transfer time under

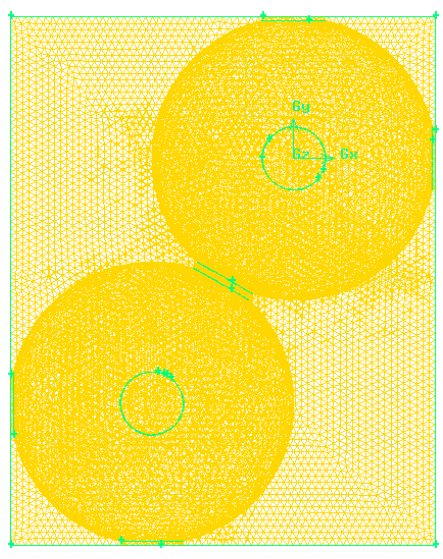

(a)

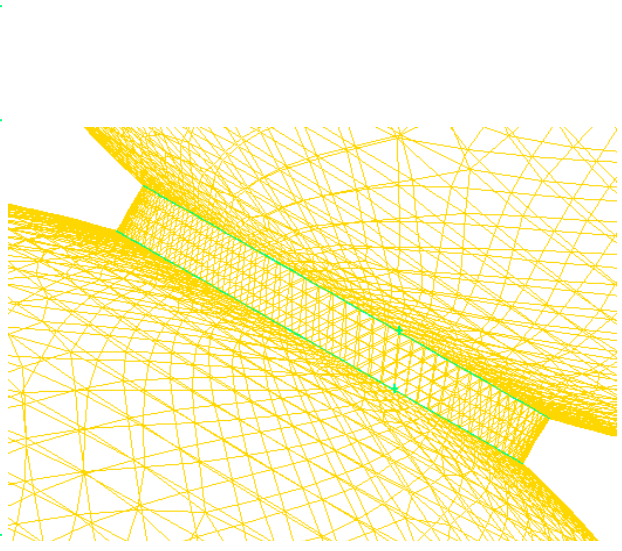

(b)

Figure 3. Overall grid and local encryption. (a) Overall grid; (b) Local encryption.

Table 1. Initial conditions.

\begin{tabular}{lcc}
\hline & Parameter name & Value \\
\hline 1 & Particle material & Ceramic ball \\
2 & Particle size & $16 \mathrm{~mm}$ \\
3 & Initial temperature & $873 \mathrm{~K}-1273 \mathrm{~K}$ \\
4 & Cooling wall temperature & $300 \mathrm{~K}$ \\
5 & Internal emissivity & 0.32 \\
6 & Surface emissivity & 0.85 \\
\hline
\end{tabular}




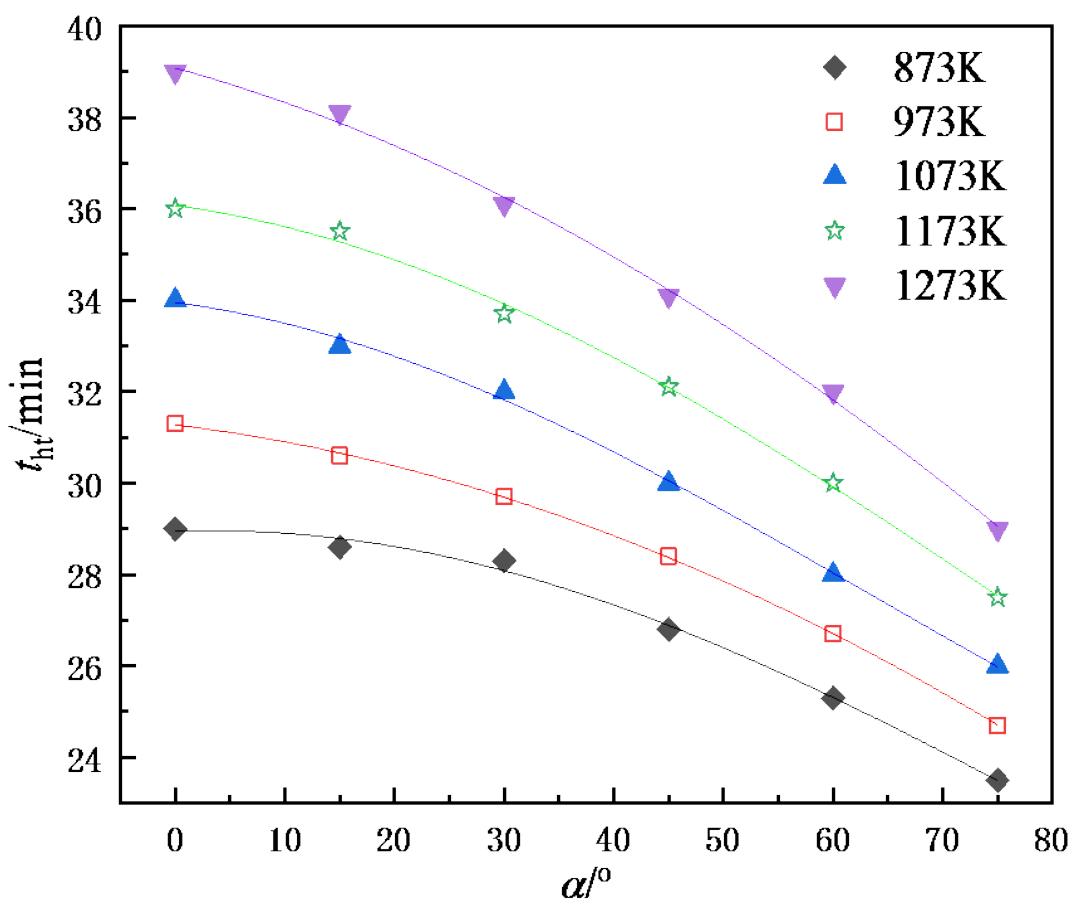

Figure 4. Effect of particle stacking angle on heat transfer time under different initial temperature conditions.

different initial temperature conditions. When particles with the same $\alpha$, the higher initial temperature causes an increase of $t_{h t}$ As $\alpha$ increases, the difference caused by different initial temperatures decreases. When initial temperature is the same, $t_{h t}$ decreases as $\alpha$ increases. The higher initial temperature causes the angle change to have a more obvious effect on $t_{h t}$. When the initial temperature is $873 \mathrm{~K}$, the increase in $\alpha$ reduces the $t_{h t}$ by $5.5 \mathrm{~min}$, and when the initial temperature is $1273 \mathrm{~K}$, the $t_{h t}$ decreases by $10 \mathrm{~min}$. The curves in the figure are all obtained by cosine function fitting, the maximum errors are all less than $2 \%$, and the correlation coefficients are all above 0.955 .

\subsection{Average Wall Heat Flux}

Figure 5 shows the average wall heat flux evolution at the initial temperature of $1073 \mathrm{~K}$. All curves can be divided into three stages: rapid decline stage (I), deceleration decline stage (II), and slow decline stage (III). The high temperature difference causes a larger heat transfer capacity and forms a larger heat transfer, particles lose heat faster and the temperature difference decreases rapidly. Thus, particles transfer a large amount of heat to cooling wall, resulting in the $\bar{q}$ rapidly decreases. The larger $\alpha$ forms the larger the $\bar{q}$, resulting in the temperature difference of particles with different $\alpha$. In the deceleration decline stage, the heat transferred to the cooling wall further decrease, the $\bar{q}$ of the particles with $\alpha=75^{\circ}$ is gradually smaller than that of the particles with other $\alpha$, and the curve appears intersections. After $9 \mathrm{~min}$, it enters the slow decline stage, the larger a causes the smaller the $\bar{q}$. The particles contain less heat, the temperature 


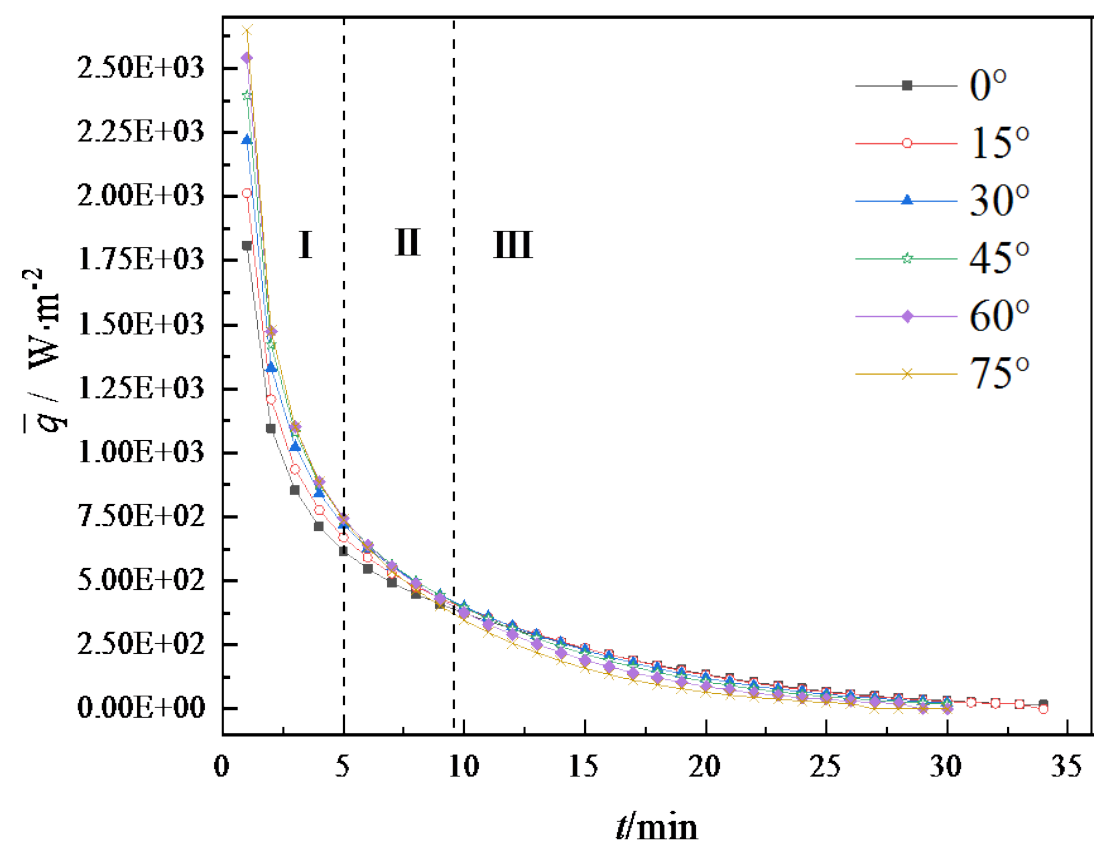

Figure 5. Average wall heat flux evolution.

difference between the particles and the cooling wall is small, and the heat transfer process is slow.

\subsection{Temperature Evolution}

Figure 6 shows the temperature distribution of the characteristic surface (the middle surface). At $5 \mathrm{~min}$, the upper and lower temperature difference of the characteristic surface is relatively large, especially $\alpha=0^{\circ}$. The larger $\alpha$ causes the lower the temperature of $\mathrm{P}_{2}$. The thermal conductivity of the particles is much greater than that of the gas phase, resulting in uneven heat transfer between the gas and solid phases. At $10 \mathrm{~min}$, the temperature of the entire characteristic surface is further reduced. Among them, $\alpha$ has a more obvious influence on $\mathrm{P}_{1}$. At $20 \mathrm{~min}, 90 \%$ of the energy contained in the particles with $\alpha=60^{\circ}$ and $\alpha=75^{\circ}$ has been transferred to cooling wall, so the overall temperature is smaller. The temperature of $\mathrm{P}_{2}$ with $\alpha=0^{\circ}$ and $\alpha=15^{\circ}$ still maintains a higher value.

Figure 7 shows the particle average temperature evolution. It shows that the larger $\alpha$ causes the lower the particle average temperature. Compared with the average wall heat flux, the average temperature changes relatively smoothly. The average temperature of the particle with $\alpha=0^{\circ}$ and $\alpha=15^{\circ}$ is not much different and the curve changes almost coincide. The maximum temperature difference of particles with different $\alpha$ appeared at $13 \mathrm{~min}$, which was about $112.4 \mathrm{~K}$. Figure 8 shows the average temperature of $\mathrm{P}_{1}$ and $\mathrm{P}_{2}$ evolution, $\alpha$ has a more obvious impact on the temperature of $\mathrm{P}_{2}$. At $1 \mathrm{~min}$, the temperature of $\mathrm{P}_{1}$ was below $900 \mathrm{~K}$, while $\mathrm{P}_{2}$ was kept at a higher temperature. A larger $\alpha$ causes an increase of the temperature drop rate. The larger the $\alpha$, the smaller the overall heat transfer distance, resulting in it easier for the heat of $\mathrm{P}_{2}$ to be transferred to cooling wall. 


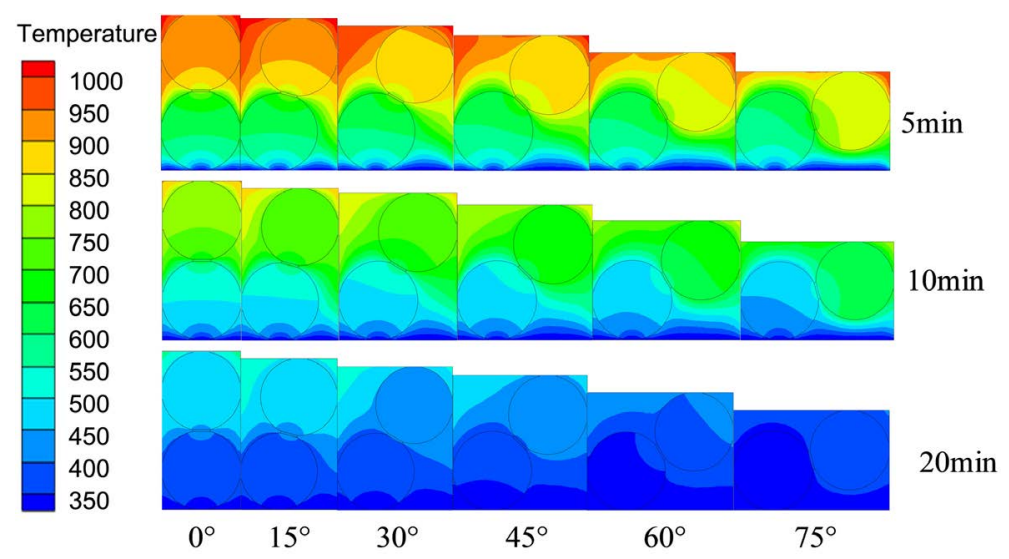

Figure 6. Temperature distribution of characteristic surface.

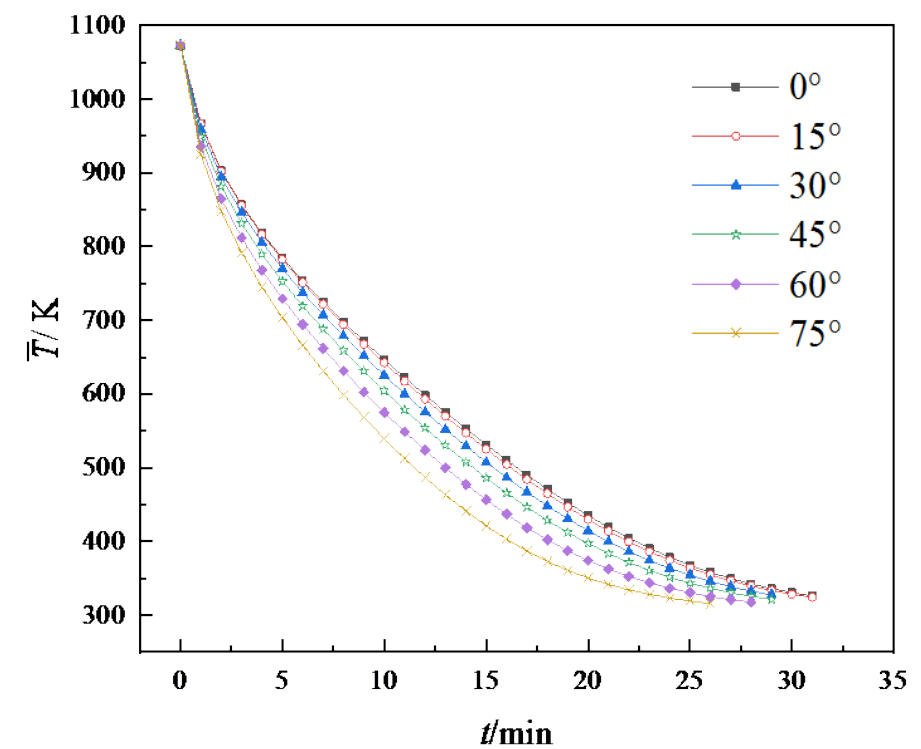

Figure 7. Particle average temperature evolution.

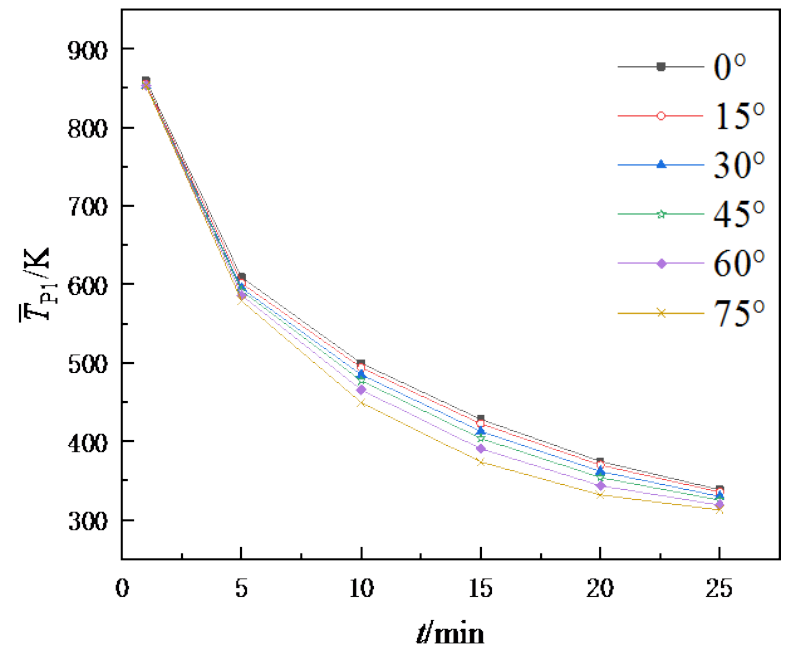

(a)

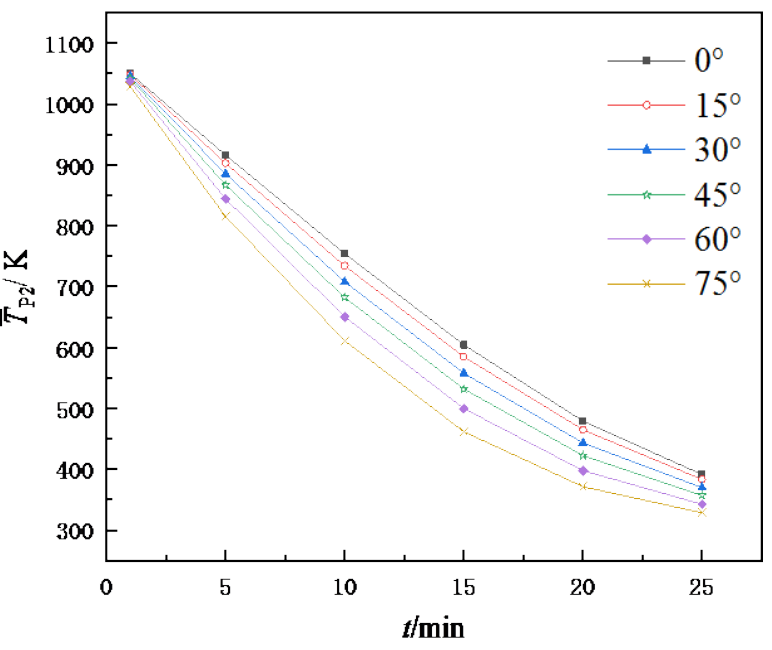

(b)

Figure 8. Temperature of $\mathrm{P}_{1}$ and $\mathrm{P}_{2}$ evolution. (a) $\mathrm{P}_{1}$; (2) $\mathrm{P}_{2}$. 


\section{Conclusions}

In this paper, an unsteady heat transfer model between two particles is established, and the effect of particle stacking angle under different initial temperature conditions on the heat transfer process of the particles close to the wall is analyzed. The major results are as follows:

1) When the initial temperature is the same, the heat transfer time decreases with the increase of $\alpha$. The higher the initial temperature causes the longer the required heat transfer time, and the more obvious the effect of particle stacking angle.

2) The average wall heat flux decreases rapidly first and then slowly decreases. First the larger stacking angle forms the greater the average wall heat flux. After $9 \mathrm{~min}$, the opposite result appeared

3) The larger the particle stacking angle causes the lower the average temperature. The change of particle stacking angle has a more obvious effect on the temperature of $\mathrm{P}_{2}$.

\section{Acknowledgements}

This work was supported by National Key R \& D Program of China (2017YFB0603504-2), and Shandong Provincial Natural Science Foundation, China (ZR2020ME176 and ZR2020ME182).

\section{Conflicts of Interest}

The authors declare no conflicts of interest regarding the publication of this paper.

\section{References}

[1] China's National Bureau of Statistics (2019). http://www.stats.gov.cn/tjsj/ndsj/2020/indexch.htm

[2] Cheng, Z.L., Guo, Z.G., et al. (2019) Waste Heat Recovery from High-Temperature Solid Granular Materials: Energy Challenges and Opportunities. Renewable and Sustainable Energy Reviews, 116, Article ID: 109428. https://doi.org/10.1016/j.rser.2019.109428

[3] Yang, J., Wang, J., et al. (2012) Experimental Analysis of Forced Convective Heat Transfer in Novel Structured Packed Beds of Particles. Chemical Engineering Science, 71, 126-137. https://doi.org/10.1016/j.ces.2011.12.005

[4] Natsui, S., Takai, H., et al. (2015) Model Study of the Effect of Particles Structure on the Heat and Mass Transfer through the Packed Bed in Ironmaking Blast Furnace. International Journal of Heat and Mass Transfer, 91, 1176-1186. https://doi.org/10.1016/j.ijheatmasstransfer.2015.08.033

[5] Zheng, B., Sun, P., et al. (2018) Heat Transfer of Calcined Petroleum Coke and Heat Exchange Tube for Calcined Petroleum Coke Waste Heat Recovery. Energy, 155, 56-65. https://doi.org/10.1016/j.energy.2018.05.013

[6] Singhal, A., Cloete, S., et al. (2017) Heat Transfer to a Gas from Densely Packed Beds of Cylindrical Particles. Chemical Engineering Science, 172, 1-12. https://doi.org/10.1016/j.ces.2017.06.003 
[7] Bu, S.S., Yang, J., et al. (2014) On Contact Point Modifications for Forced Convective Heat Transfer Analysis in a Structured Packed Bed of Spheres. Nuclear Engineering and Design, 270, 21-33. https://doi.org/10.1016/j.nucengdes.2014.01.001

[8] Beaulieu, C., Vidal, A., et al. (2020) Impact of Surface Roughness on Heat Transfer through Spherical Particle Packed Beds. Chemical Engineering Science, 231, Article ID: 116256. https://doi.org/10.1016/j.ces.2020.116256

[9] Polamuri, D. and Thamida, S.K. (2017) Simulation to Study the Effect of Natural Convection in the Voids of a 2D Granular Packed Bed of Cylindrical Particles on Effective Thermal Conductivity. Thermal Science and Engineering Progress, 2, 102-108. https://doi.org/10.1016/j.tsep.2017.05.009 